\title{
Unicornuate uterus: case reports of heterogenous presentations challenging clinical diagnosis and management
}

\author{
Swati Trivedi $^{1 *}$, Santosh Khokher ${ }^{1}$, Prasoon Rastogi $^{2}$, Vinod Kumar Dhaka ${ }^{3}$
}

\author{
${ }^{1}$ Department of Obstetrics and Gynaecology, Dr. S. N. Medical College, Jodhpur, Rajasthan, India \\ ${ }^{2}$ Department of Endocrinology, AIIMS, Jodhpur, Rajasthan, India \\ ${ }^{3}$ Department of Pediatrics, Dr. S. N. Medical College, Jodhpur, Rajasthan, India
}

Received: 19 April 2021

Accepted: 11 May 2021

\author{
*Correspondence: \\ Dr. Swati Trivedi, \\ E-mail: krishnaagnihotri.kgmc@gmail.com
}

Copyright: ( $)$ the author(s), publisher and licensee Medip Academy. This is an open-access article distributed under the terms of the Creative Commons Attribution Non-Commercial License, which permits unrestricted non-commercial use, distribution, and reproduction in any medium, provided the original work is properly cited.

\begin{abstract}
Unicornuate uterus is an anomaly arising from defective lateral fusion of incompletely developed mullerian duct or paramesonephric duct with the contralateral duct. Pregnancy in non-communicating rudimentary horn can result in I and II trimester pregnancy losses along with maternal morbidity and mortality. Here we describe three such cases of unicornuate uterus with non-communicating rudimentary horn pregnancy, who presented to our hospital with pain in lower abdomen. Two of them with II trimester pregnancy landed in haemorrhagic shock owing to rupture of pregnant horn, though were revived by immediate intervention. Third patient who came with a definitive diagnosis of I trimester rudimentary horn pregnancy was managed electively by hemi-hysterectomy. Rupture of pregnant uterus can occur in II trimester when associated with uterine anomaly. Early sonographic diagnosis has a major offering in workup, management and prevention of mother from grave life threatening consequences.
\end{abstract}

Keywords: Defective lateral fusion, Hemi-hysterectomy, Mullerian duct, Rudimentary horn, Unicornuate uterus

\section{INTRODUCTION}

The müllerian or paramesonephric duct appears on either side, lateral to each wolffian duct, approximately 37 days after fertilization and grows medio-caudally towards the urogenital sinus. They are the primordial anlage of female reproductive tract that differentiate to form the fallopian tubes, uterus, uterine cervix and upper $2 / 3 \mathrm{rd}$ of vagina. A wide variety of mullerian duct malformations result from agenesis, unilateral maturation with incomplete/absent opposite side, absent/faulty midline fusion or defective canalisation of the two mullerian ducts. According to ESHRE (European society of human reproduction and embryology) and ESGE (European society of gynecological endoscopy) classification system for female genital malformations, unicornuate uterus falls in class $\mathrm{U} 4$.
The incidence of mullerian duct anomalies is around $3.2 \%$. $^{2}$ The prevalence with imaging ranges from 0.4 to $10 \% .^{3}$ The most common anomaly found is arcuate uterus followed by septate, bicornuate, didelphic and unicornuate uterus in descending order. ${ }^{4}$ Unicornuate uterus, the least common of all occurs in 1 in 4020 women, a rudimentary horn is present in about $84 \%$ of the cases. ${ }^{2}$ Herein we report three rare cases of I and II trimester ectopic pregnancy in non-communicating rudimentary horn of unicornuate uterus.

\section{CASE REPORT}

\section{Case 1}

$\mathrm{AB}$, a 27 year old G2P1 with 4 months amenorrhoea, married for 7 years, reported in Umaid hospital, Jodhpur, in the casualty with complain of lower abdominal pain and bleeding per vaginum for 1-2 hours. She was an 
unbooked patient with no antenatal visits and had confirmed her pregnancy by self-urine pregnancy test at home. Her first pregnancy was an uneventful full term vaginal delivery 6 years back. There was no history of consanguineous marriage of parents or any abnormal pregnancies in the family.

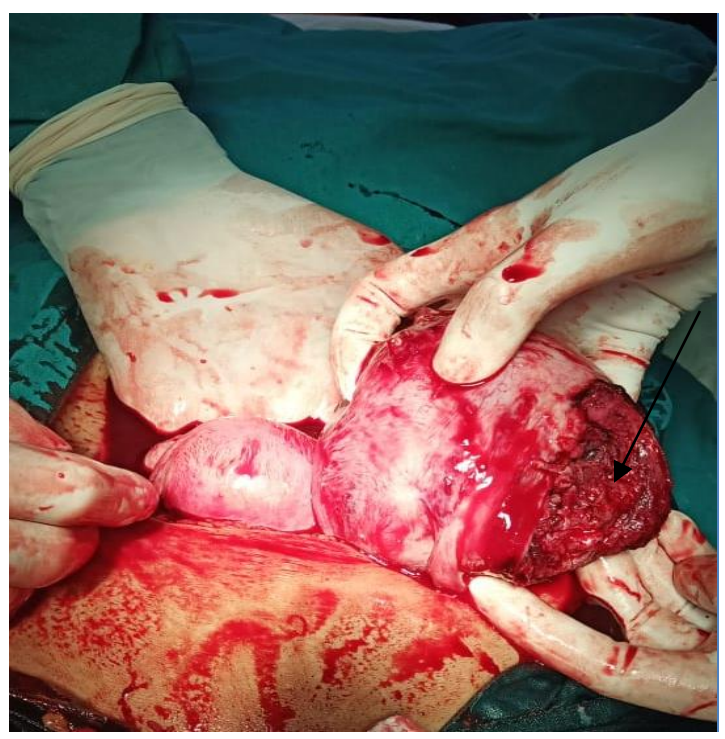

Figure 1: Unicornuate uterus with ruptured right rudimentary horn (black arrow) along the anterolateral surface.

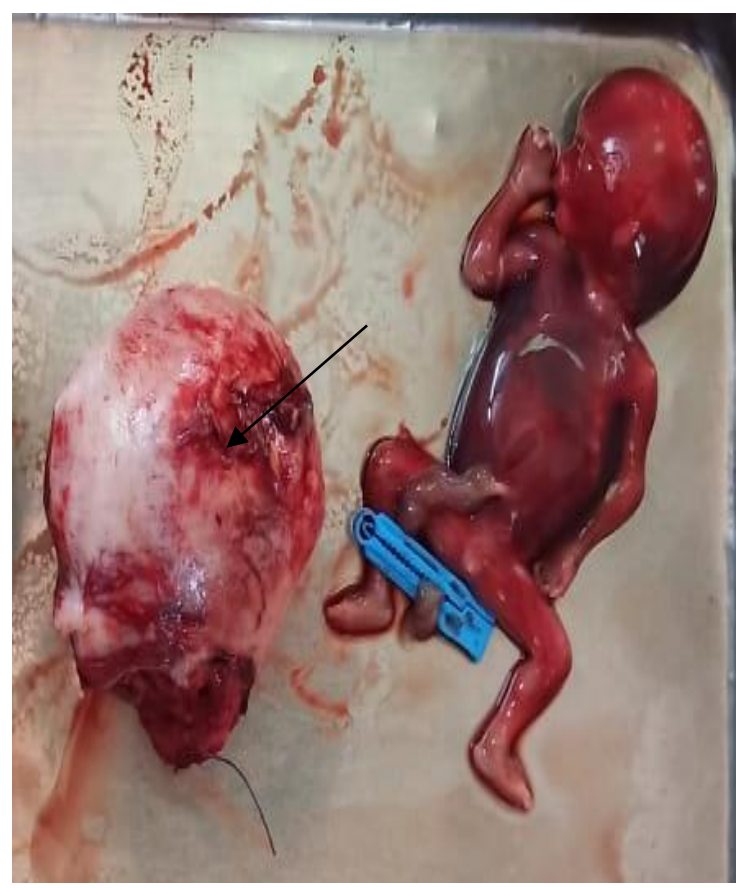

Figure 2: Gross specimen showing fetus and removed ruptured horn (black arrow) post laparotomy.

On presentation, patient's BP was 112/80 mmHg and PR was 94 /min, pallor, icterus and pedal edema were absent. Abdominal examination revealed 18-20 weeks size uterus. On per speculum, slight bleeding coming from internal os was visualised. On per vaginal examination, cervical os admits tip of finger with cervix uneffaced, uterus was soft in consistency, 16-18 weeks size. Patient was admitted and all routine blood and urine investigations were sent including a call for urgent ultrasonography (USG). After around an hour of hospital admission during emergency workup, patient started feeling dizzy and became pale. Her BP dropped to 90/60 $\mathrm{mmHg}$ and pulse rate was $120 / \mathrm{min}$. Sonography of abdomen and pelvis revealed ruptured horn of unicornuate/bicornuate uterus with fetus lying in abdominal cavity and free fluid in pelvis, probe tenderness was present. Immediately patient's resuscitation was started with IV fluids and blood requisition was sent. Urgent emergency exploratory laparotomy was decided and patient shifted to operation theatre (OT). On table, BP further fell to $70 / 40 \mathrm{mmHg}$. On exploration under general anaesthesia, gross hemoperitoneum was seen with ruptured noncommunicating rudimentary left horn of unicornuate uterus along the anterolateral surface with bleeding from the edges (Figure 1). A male dead fetus approximately 500 gms with placenta lying in peritoneal cavity was delivered followed by removal of ruptured noncommunicating horn/hemi-hysterectomy and restoration of haemostasis (Figure 2). During the whole procedure $500 \mathrm{ml}$ clots and $1000 \mathrm{ml}$ blood were suctioned from peritoneal cavity. Abdominal cavity was washed with $1000 \mathrm{ml}$ of warm normal saline followed by closure in layers. Intraoperatively one unit blood transfusion was done. Patient's vitals were stable postoperatively but there was a fall in haemoglobin from 11.4 preoperatively to 6.4 postoperatively which was corrected. Patient had a 3 days stay in gynae ICU which was uneventful, followed by discharge on day 7 with advise to use contraception for atleast a year.

\section{Case 2}

XY, a 20 year old female, 2nd gravida with previous lower segment caesarean section 4 months back (on account of non-progression of labour) came to our OPD at Umaid hospital. Patient conceived during lactational amenorrhoea and confirmed her pregnancy at home by urine pregnancy test. She had undergone an ultrasonography at some private centre on the same date, which reported a single live left adnexal ectopic pregnancy of gestational age 10 week 2 day, CRL $3.2 \mathrm{~cm}$ with fetal pole, yolk sac and cardiac activity present. Uterus $8.2 \times 3 \times 4.2 \mathrm{~cm}$ with endometrial thickness $8.6 \mathrm{~mm}$. No obvious free fluid was noted in pelvis or Morrison's pouch.

On examination, patient was conscious, alert with BP of 122/74 $\mathrm{mmHg}$, PR 78 /min, pallor, icterus and pedal edema were absent. On per abdominal examination, abdomen was soft and uterus just palpable. On per vaginum examination, uterus was soft $10-12$ weeks size. Patient was admitted and all investigations were sent along with a call for repeat USG by senior radiologist. 
Repeat USG also revealed similar findings except that pregnancy was present in rudimentary horn of uterus, bilateral ovaries were normally visualised with no free fluid in pouch of Douglas.



Figure 3: Unicornuate uterus with left non communicating rudimentary horn (black arrow) pregnancy.

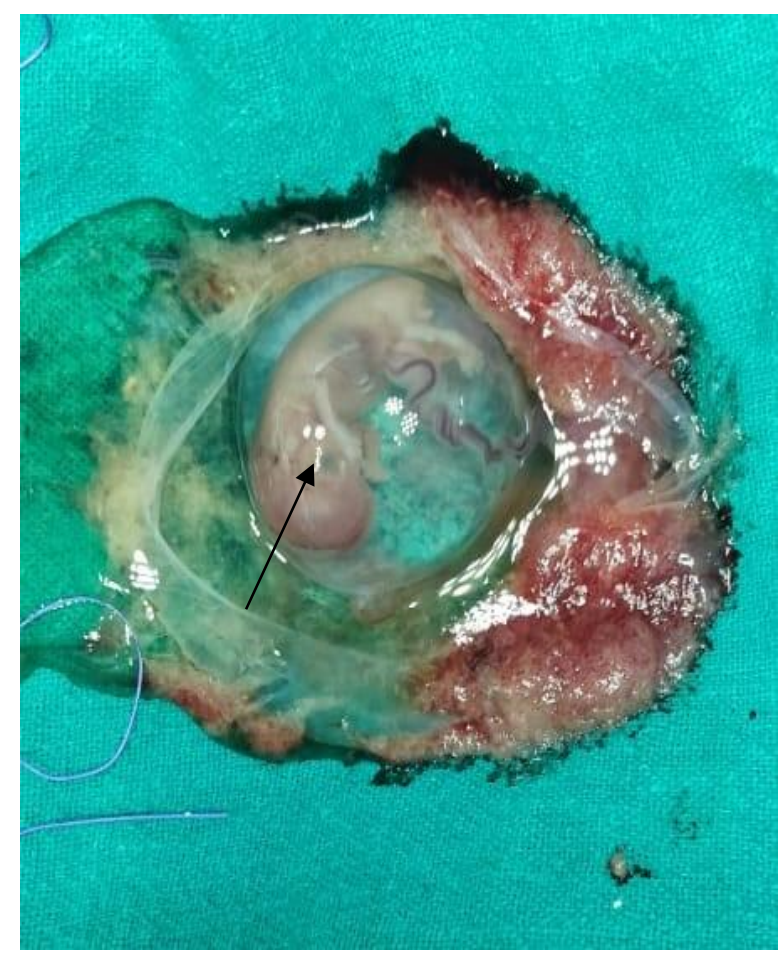

Figure 4: Intact gestational sac with 10 week fetus (black arrow) in situ delivered from unruptured rudimentary horn following hemi-hysterectomy.

Since the patient was hemodynamically stable and had no complains, we planned for elective laparotomy coming morning. Patient was shifted to operation theatre at the planned time and laparotomy was done under spinal anaesthesia. Intact left rudimentary horn with gestational sac was identified (Figure 3 and 4). It was clamped, cut and delivered out with gestational sac in situ. No communication was found with the right functional horn, hence it was a non-communicating rudimentary horn pregnancy. Patient's pre-op, intra-op and post-op periods were uneventful and was successfully discharged on day 5 of surgery.

\section{Case 3}

PQ, a 22 year primigravida with 3.5 months amenorrhoea, married for 6 months, presented to our hospital with complains of pain lower abdomen and bleeding per vaginum for 2.5 hours. She had confirmed her pregnancy by urine pregnancy test at home after missing her first period. No antenatal booking or investigations were done. On evaluation, patient was conscious with BP $116 / 78 \mathrm{mmHg}$, PR 110/min, pallor, icterus and pedal edema were absent. There was no clinical presence of pallor or icterus. On per abdominal examination, uterus was 20 weeks in size and tensetender on palpation, external ballotment could not be elicited. On per vaginal examination, cervical os was closed with slight bleeding present. Patient was admitted for workup. Sonography reported an intrauterine demise of 22 weeks with marginal hematoma of $56 \times 76 \mathrm{~mm}$. Induction of labour was done with tablet misoprostol 200 $\mu \mathrm{g}$ orally and repeated after 6 hours. 12 hours after $1 \mathrm{st}$ dose patient complained of increased pain lower abdomen. On examination abdomen was tense-tender with guarding/rigidity present, on per vaginal examination, cervical motion tenderness could be elicited, BP 100/60 mmHg, PR 140/min and clinically pallor present. A suspicion of rupture uterus arose for the confirmation of which patient was sent for urgent ultrasonography by senior radiologist, an abortus of 15.6 weeks in ruptured rudimentary horn of uterus with gross free fluid in peritoneal cavity was identified. Marginal hematoma of $9 \times 7 \mathrm{~cm}$ was noted adjacent to ruptured rudimentary horn.

Patient was immediately shifted to OT for laparotomy. Gross hemoperitoneum was present, $1500 \mathrm{ml}$ blood and almost $500 \mathrm{ml}$ clots were removed. Fetus and placenta lying in peritoneal cavity were delivered out. Left side non-communicating rudimentary horn rupture was identified, same was clamped, cut and delivered, complete haemostasis was secured (Figure 5). Left salpingectomy was also done. Right side tube and both ovaries were normal and healthy. Right unicornuate uterus was intact. Abdominal drain was placed. 2 units blood transfusion was done intraoperatively. Intraop BP 90-130/60-84 $\mathrm{mmHg}$ and PR 110-150 / min. Patient was shifted to ICU post operatively for intensive monitoring. Post-op period was uneventful. Drain was removed on day 3 of surgery and patient discharged satisfactorily on day 6 . 


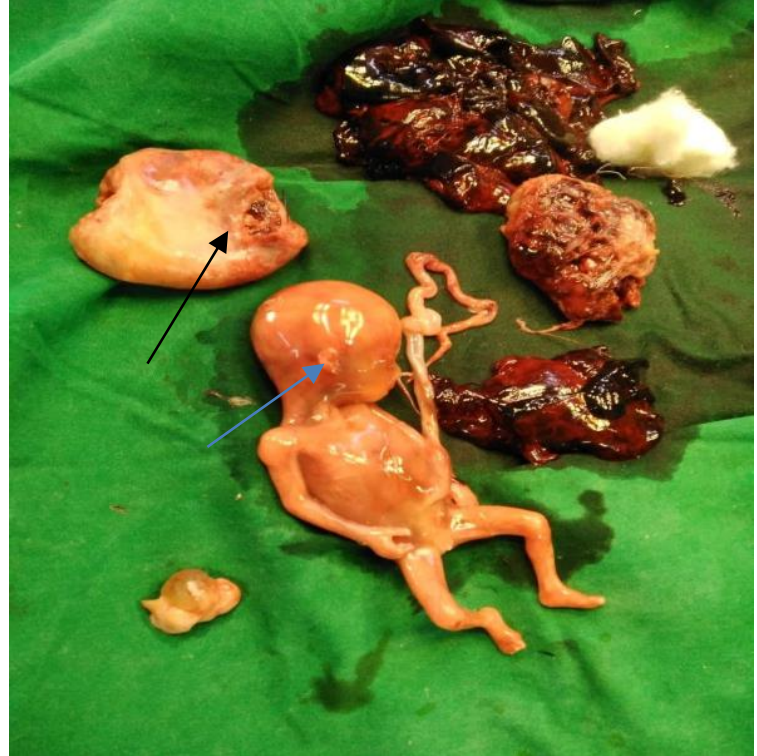

Figure 5: Gross specimen of ruptured rudimentary horn (black arrow), fetus (blue arrow) and placental tissue delivered from peritoneal cavity.

\section{DISCUSSION}

Unicornuate uterus is a disorder of symmetricunobstructed lateral fusion of mullerian ducts. A unicornuate uterus may or may not have a rudimentary horn. The rudimentary anlagen when present can communicate directly with the unicornuate uterus, although in some instances there is no cavity within the anlagen. Majority $(90 \%)$ of rudimentary horns are noncommunicating. The connections between the two could be a fibromuscular band or no connection/communication could be found between the two uterine cavities. The incidence of pregnancy in a non-communicating rudimentary horn is approximately 1 in 76,000-150,000 pregnancies and only $14 \%$ of such cases are correctly diagnosed before clinical manifestations become apparent. $^{5}$

Obstetrical risks associated with unicornuate uterus are I and II trimester miscarriages, fetal growth restriction, preterm delivery and malpresentations. Cervical incompetence, abnormal uterine blood flow, reduced size of cavity and muscle mass of the hemiuterus are proposed to underlie above risks. Rudimentary horns are at an increased risk of ectopic pregnancy within the remnant resulting from transperitoneal sperm migration which permits ovum fertilization and pregnancy. ${ }^{6}$ According to Holden and Hart, approximately 350 cases of rudimentary horn pregnancy have been reported since 1669 after the original case report by Mauriceau. ${ }^{7}$ Ruptured ectopic pregnancy of rudimentary horn can be the eventual outcome, if pregnancy is not detected early. Sudden severe intraperitoneal haemorrhage and shock, following rupture of the rudimentary horn can lead to mortality within minutes. ${ }^{8}$ The timing of rupture can vary (5 to 35 weeks) depending on the horn musculature and its ability to hypertrophy and dilate. The risk of uterine rupture is 50 to $90 \%$, with most ruptures (approximately $80 \%$ ) occurring by the end of the second trimester. ${ }^{9}$ Bleeding from the ruptured rudimentary horn is severe in pregnancy because of increased thickness and vascularity of uterine wall. ${ }^{5}$ As happened in our 1 st case description where patient deteriorated hemodynamically within an hour of admission due to rupture of rudimentary horn. Whereas in our 3rd case, the patient misdiagnosed as IUD, while attempting termination went into shock owing to rupture. With uterine rupture, urgent intervention should be done (case 1 and 3). Excision of the rudimentary horn with ipsilateral salpingectomy should be performed without delay in hemodynamically stable patients. ${ }^{10}$

It is yet unexplained why some patients with a uterine anomaly have normal reproductive function, while others abort in early pregnancy. As in our 1st and 2nd case, both patients had a previous term pregnancy (which possibly must have been present in main functional horn). A case reported by Nwosu and co-workers of a woman who had had one previous normal pregnancy but developed ruptured uterus in her second pregnancy. ${ }^{11}$ Though it has been reported that the chance for a liveborn child increases with each pregnancy loss, yet it is undivulged whether this apparent conditioning of the uterus is due to better vascularisation or better myometrial stretching and accommodation or some other factor. A medical history of $>3$ episodes of spontaneous abortion or premature labour merits evaluation of the uterine cavity for structural abnormalities. An abnormality is found in approximately $10 \%$ of such cases, in which scenario, removal of rudimentary horn should be done. This increases the chances of a successful pregnancy by eliminating the risk of ectopic pregnancy in rudimentary horn as well as the possibility of retrograde menstruation leading to endometriosis and infertility. ${ }^{12}$ Since in our case report there was no such history, we ended up doing laparotomy for ruptured rudimentary horn.

Mullerian anomalies are usually asymptomatic and discovered accidently during pelvic examination, caesarean section, tubal sterilization or infertility evaluation. Depending on the clinical presentation, diagnostic modalities included USG, hysterosalpingography (HSG), MRI, hysteroscopy and laparoscopy. Sonography in I trimester has an increased chance of identifying uterine anomaly or pregnancy in rudimentary horn.

\section{CONCLUSION}

Diagnosis of mullerian anomalies remains a challenge. Yet if identified timely before any complications or rupture (diagnostic modality of choice-USG), we can prevent maternal morbidity and mortality to quite an extent. This holds more of an importance for developing countries where most pregnancies are unsupervised and unbooked till the time of delivery nears or till any 
complications develop. Moreover, patients with such anomalies should be vigilantly counselled both antenatally and postnatally before discharge, regarding contraception practices and that their next pregnancy should be a planned one with supervision since the very beginning.

Funding: No funding sources Conflict of interest: None declared

Ethical approval: Not required

\section{REFERENCES}

1. Grimbizis GF, Gordts S, Sardo ADS, Brucker S, Angelis CD, Gergolet M, et al. The ESHRE/ESGE consensus on the classification of female genital tract congenital anomalies. Hum Reprod. 2013;28(8):2032-44.

2. Reichman D, Laufer MR, Robinson BK. Pregnancy outcomes in unicornuate uteri: a review. Fertil Steril. 2009;91(5):1886-94.

3. Troiano RN, McCarthy SM. Müllerian duct anomalies: imaging and clinical issues. Radiology. 2004;233(1):19-34.

4. Grimbizis GF, Camus M, Tarlatzis BC, Bontis JN, Devroey P. Clinical implications of uterine malformations and hysteroscopic treatment results. Hum Reprod Update. 2001;7(2):161-74.

5. Arslan T, Bilgiç E, Sentürk MB, Yücel $N$. Rudimentary uterine horn pregnancy: a mystery diagnosis. Fertil Steril. 2009;92(6):2037.
6. Buntugu K, Ntumy $\mathrm{M}$, Ameh $\mathrm{E}$, Obed $\mathrm{S}$. Rudimentary horn pregnancy: pre-rupture diagnosis and management. Ghana Med J. 2008;42(2):92-4.

7. Holden R, Hart P. First-trimester rudimentary horn pregnancy: prerupture ultrasound diagnosis. Obstet Gynecol. 1983;61(3):56-8.

8. Dogra Y, Minhas S, Marwaha PD. Müllerian duct anomaly with congenital rectovaginal fistula: a rare case presentation. South Afr J Obstet Gynaecol. 2015;21(1):22.

9. Rackow BW, Arici A. Reproductive performance of women with müllerian anomalies. Curr Opin Obstet Gynecol. 2007;19(3):229-37.

10. George Pados G, Tsolakidis D, Athanatos D, Almaloglou K, Nikolaidis N, Tarlatzis B. Reproductive and obstetric outcome after laparoscopic excision of functional, noncommunicating broadly attached rudimentary horn: a case series. European J Obstet Gynecol Reproductive Bio.2014;182:33-7.

11. Nwosu BO, Ugboaja JO, Obi-Nwosu A. Spontaneous rupture of gravid horn of bicornuate uterus at term: a case report. Niger Med J. 2010;51(4):184-5.

12. Panayotidis C, Prabhu S. Management of unruptured pregnancy in a rudimentary horn of unicornuate uterus at 14 weeks gestation. Gynecol Surg. 2007;4(4):281-4.

Cite this article as: Trivedi $\mathrm{S}$, Khokher $\mathrm{S}$, Rastogi $\mathrm{P}$, Dhaka VK. Unicornuate uterus: case reports of heterogenous presentations challenging clinical diagnosis and management. Int J Reprod Contracept Obstet Gynecol 2021;10:2514-8. 